\title{
Bandgap Engineering of Double Perovskites for One- and Two-photon Water Splitting
}

\section{Castelli, Ivano Eligio; Thygesen, Kristian Sommer; Jacobsen, Karsten Wedel}

\section{Published in:}

Materials Research Society Symposium Proceedings

Link to article, DOI:

10.1557/opl.2013.450

Publication date:

2013

Link back to DTU Orbit

Citation (APA):

Castelli, I. E., Thygesen, K. S., \& Jacobsen, K. W. (2013). Bandgap Engineering of Double Perovskites for Oneand Two-photon Water Splitting. Materials Research Society Symposium Proceedings, 1523, 18-23. https://doi.org/10.1557/opl.2013.450

\section{General rights}

Copyright and moral rights for the publications made accessible in the public portal are retained by the authors and/or other copyright owners and it is a condition of accessing publications that users recognise and abide by the legal requirements associated with these rights.

- Users may download and print one copy of any publication from the public portal for the purpose of private study or research.

- You may not further distribute the material or use it for any profit-making activity or commercial gain

- You may freely distribute the URL identifying the publication in the public portal 


\section{Bandgap Engineering of Double Perovskites for One- and Two-photon Water Splitting}

Ivano E. Castelli ${ }^{1}$ and Kristian S. Thygesen ${ }^{1}$ and Karsten W. Jacobsen ${ }^{1}$

${ }^{1}$ Center for Atomic-scale Materials Design, Department of Physics, Technical University of

Denmark, Kongens Lyngby, DK-2800, Denmark.

\section{ABSTRACT}

Computational screening is becoming increasingly useful in the search for new materials. We are interested in the design of new semiconductors to be used for light harvesting in a photoelectrochemical cell. In the present paper, we study the double perovskite structures obtained by combining 46 stable cubic perovskites which was found to have a finite bandgap in a previous screening-study. ${ }^{1}$ The four-metal double perovskite space is too large to be investigated completely. For this reason we propose a method for combining different metals to obtain a desired bandgap. We derive some bandgap design rules on how to combine two cubic perovskites to generate a new combination with a larger or smaller bandgap compared with the constituent structures. Those rules are based on the type of orbitals involved in the conduction bands and on the size of the two cubic bandgaps. We also see that a change in the volume has an effect on the size of the bandgap. In addition, we suggest some new candidate materials that can be used as photocatalysts in one- and two-photon water splitting devices.

\section{INTRODUCTION}

Density Functional Theory (DFT) is a powerful method to investigate structural and electronic properties of materials. The enormous increase of computational power in combination with theory developments, like the implementation of new, more reliable exchangecorrelation functionals, have made it possible to search for new materials using ab-initio quantum mechanical calculations involving several thousands of simulations. Many efforts we have been recently made to design new materials using computational tools, for example screening for organic photovoltaics, ${ }^{2-3}$ inorganic scintillators ${ }^{4}$ and bandgap engineering. ${ }^{1,5-7} \mathrm{In}$ previous works, ${ }^{1,6}$ we addressed one of the most pressing problems of our time, i.e. the development of sustainable energy technology, focusing on the photoelectrochemical conversion of water into hydrogen and oxygen using visible solar light considering both the one- and twophoton water splitting processes. Out of 19000 materials in the cubic perovskite structure, obtained by combining 52 interesting metals with oxygen, nitrogen, sulfur and fluorine, we suggested 20 combinations for one-photon water splitting and 12 additional ones for the twophoton process.

In this work, we consider the double perovskite structure for finding new rules to combine metals to obtain the desired bandgap size and to predict new candidates for water splitting. In the previous study we identified 46 stable $\mathrm{ABO}_{3}$ perovskites with a non-vanishing bandgap and here we look at combinations of those in the so-called double perovskite structure shown in Figure 1A. The chemical formula is $\mathrm{A}_{1} \mathrm{~A}_{2} \mathrm{~B}_{1} \mathrm{~B}_{2} \mathrm{O}_{6} ; \mathrm{A}_{1}$ and $\mathrm{A}_{2}$ have an oxygen coordination of 12 and are in general of larger radius than the $B_{1}$ and $B_{2}$ ions where the coordination is only 6 . The unit cell is composed of 20 atoms. Double perovskites have been first synthesized in $1998^{8}$ and they have been investigated theoretically because of their various properties, like ferroelectricity and high magnetic Curie temperature ${ }^{9}$ or small bandgap. ${ }^{10}$ 


\section{THEORY}

A material should at least fulfill three main requirements to be a good photocatalyst for water splitting: (i) structural and chemical stability against oxidation, (ii) optimal bandgap to absorb the visible part of the solar spectrum, and (iii) well positioned band edges with respect to the oxygen and hydrogen evolution potentials. In addition, the electrons and the holes should show a good mobility to reach the surface before recombining, and to be an eco-friendly material, the combination should not contain expensive or toxic chemical elements. We screen only for (i), (ii), and (iii), i.e. we search for a stable semiconductor with well positioned band edges with respect to the water redox potential. The screening is performed using the DFT-code GPAW. ${ }^{11-12}$ All the structures under investigation are fully relaxed using a standard DFT-GGA exchange-correlation functional (RPBE-approximation ${ }^{13}$ ) and the formation energies are calculated with respect to a pool of reference systems in which the material can be decomposed using a linear programming method. The pool contains the single- and bi-metal oxides as well as single metal bulks in their most stable structure as present in the experimental ICSD and in the Materials Project databases. ${ }^{14-15}$ The chemical potential for oxygen is taken relative to water because the material will be working in an aqueous environment. The bandgaps are obtained using the GLLB-SC exchange-correlation potential, ${ }^{16}$ that includes the calculation of the derivative discontinuity and gives reliable results compared to experiments. ${ }^{1}$ The evaluation of the positions of the band edges in principle requires the construction of a surface and a reliable modeling of the water layer on it. We do not use this approach because it is computationally expensive. More recently, Wu et al. ${ }^{17}$ have proposed a method for evaluating the positions of the band edges in three steps that involve two bulk calculations (the material and water) and an interfacial slab. This method has also been used to propose new oxynitrides to be used as photocatalyst in a water splitting device. ${ }^{7}$ Instead, here we use an empirical equation that gives the position of the center of the bandgap as the geometrical average of the electronegativities in the Mulliken's scale of the chemical constituents. The position of the edges is thus obtained adding and subtracting half of the bandgap. ${ }^{18}$ This approach has been validated and extensively used in our previous papers. 1,6

\section{RESULTS}

Using the method described above, we calculate the heats of formation and the bandgaps for the double perovskites under investigation. Almost all the double perovskites are stable (within a threshold we use of $0.2 \mathrm{eV}$ per atom) when compared to the existing structures into which the material can be split apart. The double perovskite maintains the structural characteristic of the two $\mathrm{ABO}_{3}$ perovskites and the major improvement in the stability is given by a possible octahedral tilting of the oxygen atoms that cannot appear in the small 5 atoms unit cell used for the cubic perovskite.

Figure 1B reports the bandgaps for all the combinations investigated. The bandgaps are seen to span over a region from 0 to more than $7 \mathrm{eV}$. The cubic perovskites along the two axes of the plot are sorted using a cluster analysis, which brings two compounds close to each other if they behave similarly with respect to bandgap formation. ${ }^{19}$ Most of the combinations investigated are seen to be insulators with a bandgap larger than $4 \mathrm{eV}$ and rather few have bandgaps in the visible range. 
A)

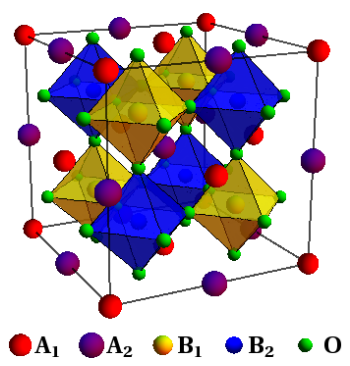

C)

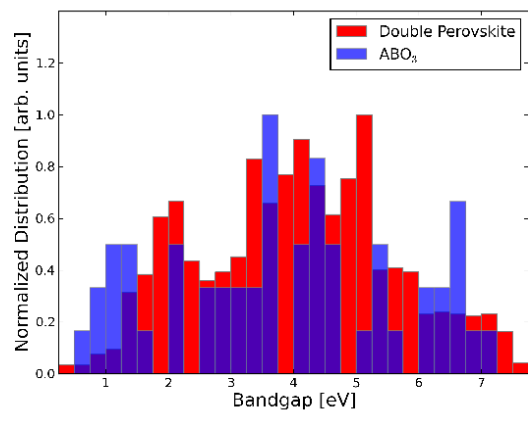

B)

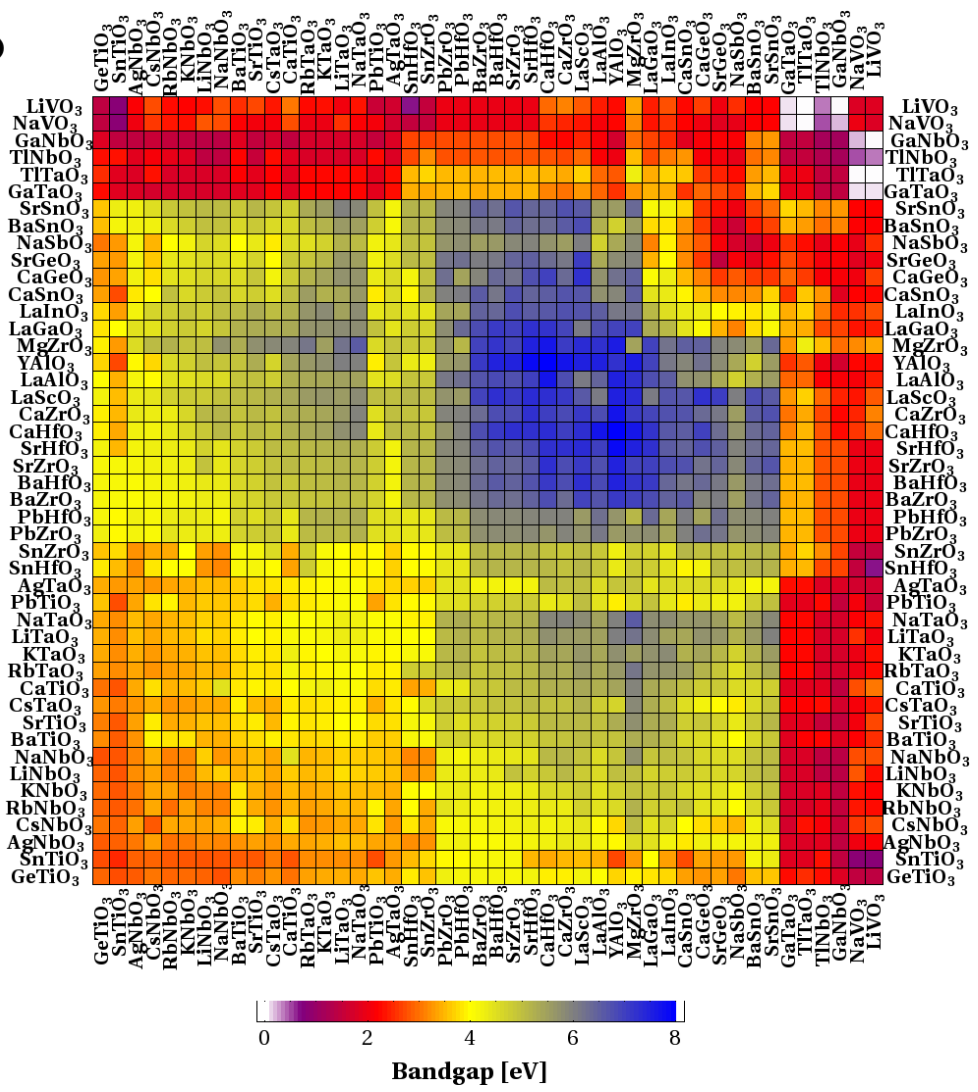

Figure 1: A) Double perovskite crystal structure. B) Calculated bandgaps for all the possible combinations. The ideal bandgap for one-photon water splitting is the red region. $C)$ Normalized distribution of the calculated bandgaps for the $\mathrm{ABO}_{3}$ (in blue) and double perovskite (in red) structures.

The normalized distributions of the bandgaps for the cubic and double perovskites are shown in Figure 1C. The distribution of the double perovskite bandgaps is less fluctuating than the one for the cubic perovskites simply because of the larger space under investigation. In fact, we are considering around 1100 double perovskite and only 46 cubic ones. In general, there is not much difference between the two distributions: both structures have a high tendency for forming large bandgap insulators and do not exhibit many compounds with a bandgap in the visible range. In the region between $0.5 \mathrm{eV}$ and $1.5 \mathrm{eV}$ there seems to be relatively few double perovskite while there seems to be a bit more in the region between $1.5 \mathrm{eV}$ and $2.5 \mathrm{eV}$. This might be caused by the octahedral distortion that leads by itself to an increase of the bandgap. The 5 atoms unit cell used to model the cubic perovskite does not allow the system to show the octahedral tilting.

In Figure 2A we show the calculated difference between the bandgaps obtained for the double perovskites and the average of the two bandgaps of the constituents $\mathrm{ABO}_{3}$ (calculated for the 20 atoms unit cell). Most of the double perovskites have a bandgap close to the average gap of the constituent structures (in white). $\mathrm{ABO}_{3}$ materials with small bandgaps (below $1.5 \mathrm{eV}$ ) show a general reduction of the bandgap (in blue). However, there are also combinations for which there is a significant increase of the bandgap (up to $3 \mathrm{eV}$, in red). This happens when we 
combine a d-metal with a p-metal in the two B-ion positions. In contrast with this, the elements in the A-ion positions do not affect the size of the resulting bandgap very much.
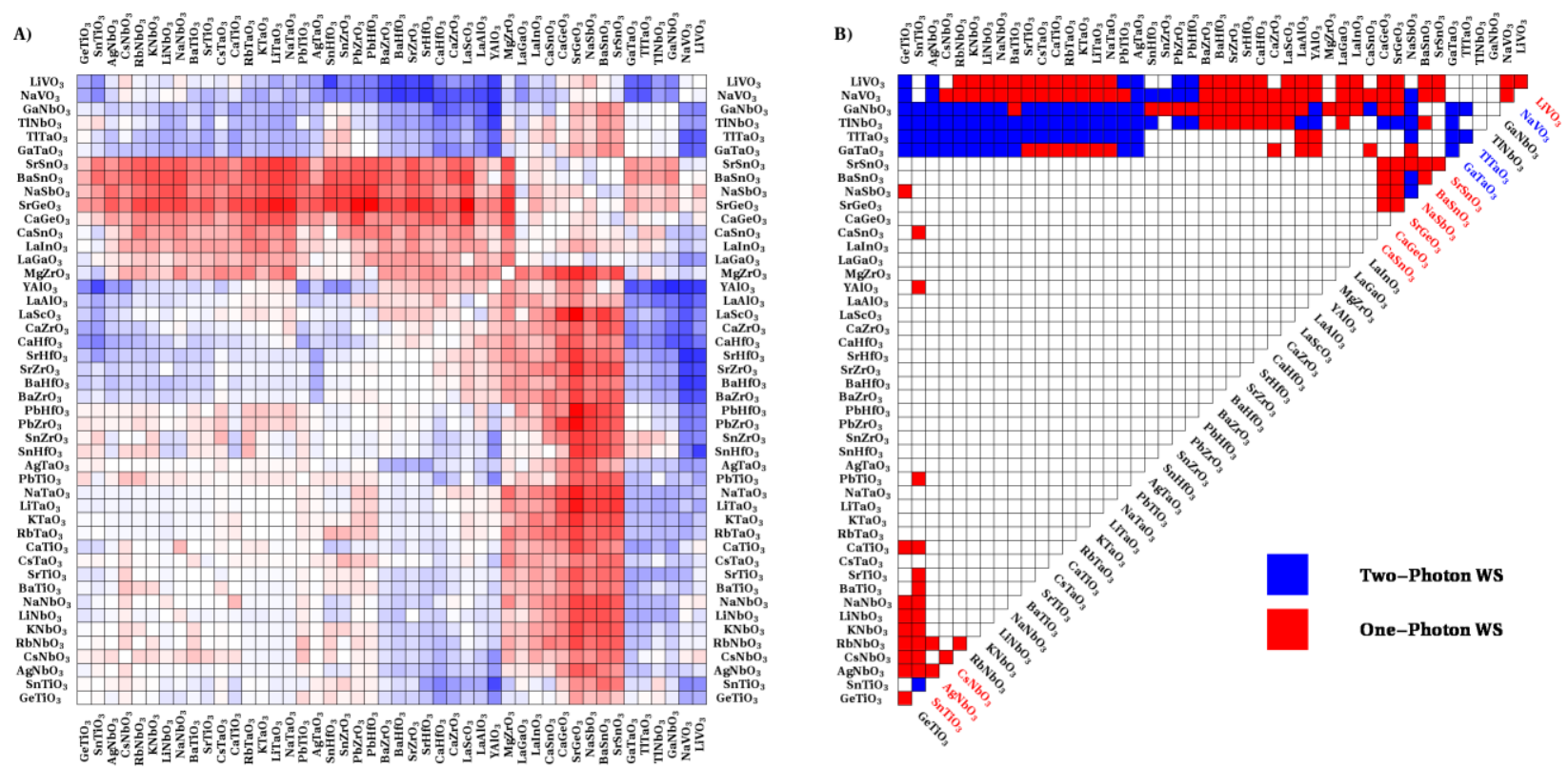

Figure 2: A) Difference between the double perovskite bandgap and the average gaps of the constituent perovskites. B) Candidates for one- and two- photon water splitting. The colored font for the name of the structure indicates that that cubic perovskite was already found as a candidate for one- or two- photon water splitting.

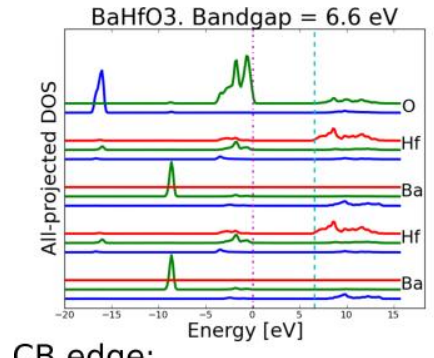

CB edge:

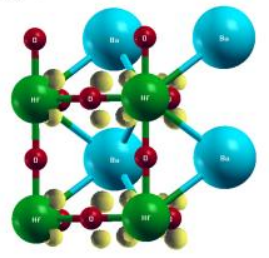

$\mathrm{BaHfO}_{3}$
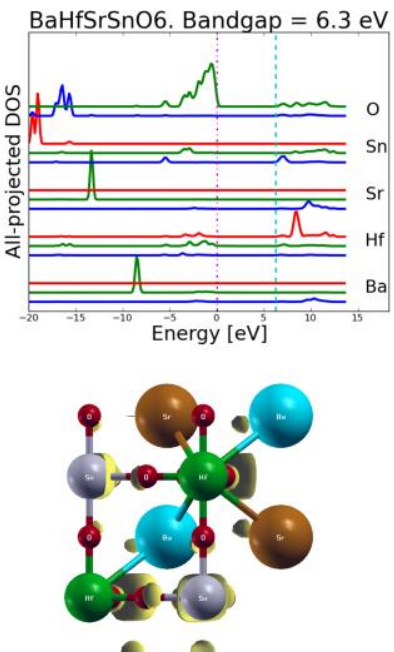

$\mathrm{BaHfSrSnO}_{6}$
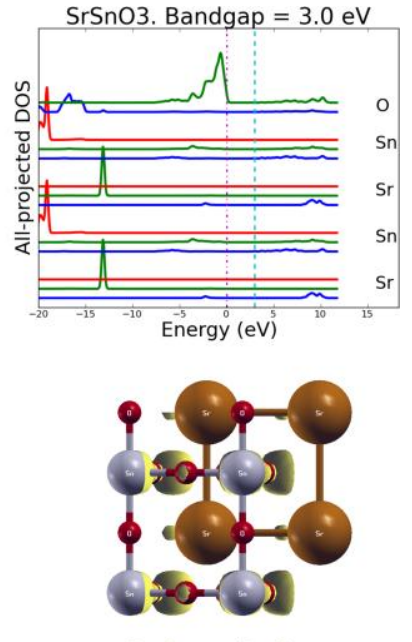

$\mathrm{SrSnO}_{3}$

Figure 3: Projected density of states for $\mathrm{BaHfO}_{3}, \mathrm{BaHfSrSnO}_{6}$, and $\mathrm{SrSnO}_{3}$. The s, p, and d orbitals are drawn in blue, green, and red, respectively. The size of the bandgap, the conduction band edges (HOMO, in magenta) and the valence band edges (LUMO, in cyan) are also indicated. The zero energy is set to correspond to the HOMO level. The increase in the bandgap for the combined perovskite is due to a strong hybridization between the $p$ - and d-metals in the $B$-ion positions. The wavefunctions of the conduction band (CB) edge states of the three combinations are also plotted. 
The reason for the opening of the bandgap compared to the average value is a strong hybridization between the p- and d-metals in the B-ion positions as shown in Figure 3 where we plot the projected density of states (DOS) for three systems: $\mathrm{BaHfO}_{3}, \mathrm{SrSnO}_{3}$, and their combination $\mathrm{BaHfSrSnO}_{6}$. The projected DOS for the Hf-d and Sn-s/p orbitals change when considering the two separated structures compared to the double perovskite and they show peaks, in particularly $\mathrm{Hf}$ and $\mathrm{Sn}$, in the double perovskite that are not present in the cubic ones. The conduction band wavefunctions for the systems follow the densities: the d-like wavefunction present around $\mathrm{Hf}$ in the $\mathrm{BaHfO}_{3}$ disappears in favor of a more intense s-like wavefunction around $\mathrm{Sn}$ in the $\mathrm{BaHfSrSnO}_{6}$. The highest occupied molecular orbital (HOMO) is in general formed by oxygen $2 p$ states and the lowest unoccupied molecular orbital (LUMO) by states of the B-ions. These features are typical of the perovskite oxides.

\section{Candidates for One- and Two-photon Water Splitting}

On top of analyzing the bandgap formation, we screen for two applications: one- and two-photon water splitting. In the one-photon water splitting, a photon is absorbed by a single semiconductor and creates an electron-hole pair. The electron and hole reach two different points of the surface and evolve hydrogen and oxygen, respectively. In the two-photon water splitting, two materials and two photons are needed for the evolution of $\mathrm{H}_{2}$ and $\mathrm{O}_{2}$. Even if two photons are required, the efficiency of a tandem device can be higher than the one obtained for the onephoton device because of different bandgap windows under consideration. In fact, to run the onephoton reaction a bandgap between 1.5 and $3 \mathrm{eV}$ is required, while for the two-photon water splitting, the optimal bandgap combination is 1.1 and $1.7 \mathrm{eV}$. In our screening, we consider silicon as the $1.1 \mathrm{eV}$ bandgap semiconductor for the evolution of hydrogen, in combination with a material with a bandgap in the range 1.3 - $3 \mathrm{eV}$ responsible for the oxygen evolution. In the one-photon water splitting the band edges should straddle the redox potentials of water. For the two-photon device, the valence band of the cathode should be above the conduction band of the anode to let the electron move from the semiconductor to silicon and combine with the hole in order to keep the charge neutrality. More detailed explanations can be found in Ref. 7.

The outcome of the screening is shown in Figure 2B. We find around 150 and 100 combinations for one- (in red) and two-photon (in blue) water splitting, respectively. 6 cubic perovskites are particularly good to generate candidates independently of the other structure. As described before, the unit cell is composed of 20 atoms. The resulting candidates for the screening of the cubic perovskite oxides are shown with colored fonts in the diagonal of Figure 2B. Comparing the results obtained for the 5 and the 20 atoms unit cell, some combinations now fall in or out of the interesting window for the one- or two-photon water splitting because of distortions of the structure. This is the case, for example, of $\mathrm{CaGeO}_{3}$ or $\mathrm{CaSnO}_{3}$, for which the most common structure is the orthorhombic perovskite with a significant octahedral tilting followed by an increase of the bandgap that is now outside the visible range window, i.e. larger than $3 \mathrm{eV}$. For some combinations the bandgap changes compared with the one calculated with the 5 atoms unit cell because of a different lattice parameters. In general, the size of the bandgap can be tuned applying a strain to the cell. This happens for $\mathrm{GeTiO}_{3}$ and $\mathrm{RbNbO}_{3}$, for example, where the bandgaps narrow and lie in the visible range so that they might be used as light harvesting materials. 


\section{CONCLUSIONS}

In this work, we have applied a screening approach to investigate the electronic properties of semiconductors in the double perovskite structure. We have focused on the problem of light harvesting and of solar light conversion into hydrogen and oxygen. We have found a general rule for combining elements to obtain a desired bandgap: two combinations with only por d-metals in the B-ion positions show bandgaps close to the average gaps of the constituents, while the combination of a p- and a d-metal results in an increase of the double perovskite bandgap. The hybridization of the orbitals of the two A-ions is very small, so it has only little influence on the change of the bandgap. A change in the volume is also influencing the size of the bandgap. We have also proposed some combinations that potentially can be used as light harvesting materials and that requires for a more thorough theoretical and experimental investigation.

\section{ACKNOWLEDGMENTS}

The authors acknowledge support from the Danish Center for Scientific Computing through grant HDW-1103-06, from the Catalysis for Sustainable Energy (CASE) initiative funded by the Danish Ministry of Science, Technology and Innovation, and from the Center on Nanostructuring for the Efficient Energy Conversion (CNEEC) at Stanford University.

\section{REFERENCES}

1. I.E. Castelli, T. Olsen, S. Datta, D.D. Landis, S. Dahl, K.S. Thygesen and K.W. Jacobsen, Energy Environ. Sci., 2012, 5, 5814.

2. R. Olivares-Amaya, C. Amador-Bedolla, J. Hachmann, S. Atahan- Evrenk, R.S. Sanchez-

Carrera, L. Vogt and A. Aspuru-Guzik, Energy Environ. Sci., 2011, 4, 4849.

3. N.M. O’Boyle, C.M. Campbell and G.R. Hutchison, J. Phys. Chem. C, 2011, 115, 16200.

4. R. Armiento, B. Kozinsky, M. Fornari and G. Ceder, Phys. Rev. B, 2011, 84, 14103.

5. M. d'Avezac, J.-W. Luo, T. Chanier and A. Zunger, Phys. Rev. Lett., 2012, 108, 027401.

6. I.E. Castelli, D.D. Landis, K.S. Thygesen, S. Dahl, I. Chorkendorff, T.F. Jaramillo, and K.W. Jacobsen, Energy Environ. Sci., 2012, 5, 9034.

7. Y. Wu, P. Lazic, G. Hautier, K. Persson and G. Ceder, DOI: 10.1039/C2EE23482C.

8. K.-I. Kobayashi, T. Kimura, H. Sawada, K. Terakura and Y. Tokura, Nature, 1998, 395, 677.

9. Z.W. Song, and B.G. Liu, arXiv:1210.5981

10. R.F. Berger and J.B. Neaton, arXiv:1209.2187

11. J.J. Mortensen, L.B. Hansen and K.W. Jacobsen, Phys. Rev. B, 2005, 71, 35109.

12. J. Enkovaara, et al., J. Phys.: Condens. Matter, 2010, 22, 253202.

13. B. Hammer, L.B. Hansen and J.K. Nørskov, Phys. Rev. B, 1999, 59, 7413.

14. ICSD database, http://www.fiz-karlsruhe.de/icsd_web.htm

15. Materials Project - A Materials Genome Approach, http://materialsproject.org/

16. O. Gritsenko, R. van Leeuwen, E. van Lenthe and E.J. Baerends, Phys. Rev. A, 1995, 51, 1944.

17. Y. Wu, M.K.Y. Chan, and G. Ceder, Phys. Rev. B, 2011, 83, 235301 .

18. M.A. Butler and D.S. Ginley, Journal of The Electrochemical Society, 1978, 125, 228.

19. I.E. Castelli, K.S. Thygesen and K.W. Jacobsen, in preparation. 